\title{
The Implications of Second-Order Functional Derivative Convergence for Adaptive Finite-Element Electromagnetics
}

\author{
Steve McFee and Dennis Giannacopoulos
}

\begin{abstract}
The usefulness of second-order functional derivatives for resolving convergence properties in adaptive finite-element electromagnetics is investigated. Second-order criteria are proposed to assess the convergence of the global solution for arbitrary discretizations of scalar Helmholtz systems. Effective convergence criteria are introduced and evaluated for some practical applications.
\end{abstract}

Index Terms-Adaptive systems, electromagnetic analysis, error analysis, finite-element methods.

\section{INTRODUCTION}

$\mathbf{T}$ HE STUDY of refinement criteria for finite-element adaption in electromagnetics has been the focus of a considerable amount of work for more than a decade and is now a well-established research area [1], [2]. Today, a variety of local error indicators are used in practice for establishing reliable solution error distributions, which can then be efficiently corrected by selectively adding degrees of freedom (DOF) to regions of high relative error [3], [4]. In addition, the study of the convergence of global solution accuracy for finite-element electromagnetics has similarly received substantial attention [5], [6]. It is now widely accepted that the rate of solution convergence is strongly dependent on both the specific problem under consideration and the type of adaption model used [7]. For example, it has been demonstrated that the intensity of local singularities in the field solution, as well as the percent increment in the number of DOF used in each adaptive step to update a discretization, can significantly affect the convergence rate of a given adaptive refinement scheme [2], [5], [7]. Consequently, for adaptive refinement strategies applied to practical systems, it is common to estimate the solution accuracy by using $a$ posteriori error estimators. Typically, these are either identical or closely related to the error indicators used to guide the adaptive process [1]-[4]. However, in this work, it is demonstrated that local error estimators can fail to effectively resolve the convergence properties of finite-element solutions under certain conditions. The purpose of this paper is to investigate the usefulness of second-order functional derivative-based criteria for assessing the convergence of the global solution accuracy more reliably for arbitrary discretizations of scalar Helmholtz systems. The essential components of the underlying derivation are discussed in the next section.

Manuscript received July 5, 2001. This work was supported in part by the Natural Sciences and Engineering Research Council of Canada.

The authors are with the Department of Electrical and Computer Engineering, McGill University, Montreal, QC, H3A 2A7 Canada.

Publisher Item Identifier S 0018-9464(02)00894-4.

\section{SECOND-ORDER FunCtional DERIVATIVES}

Functional derivative based local error indicators have been previously developed and employed successfully in adaptive finite-element methods for electromagnetics [4], [8], [9]. However, the objective of this contribution is to study the convergence properties of second-order functional derivatives with respect to finite-element global solution accuracy in scalar Helmholtz systems. Therefore, it is appropriate to first define the specific functional that will be considered, as well as the functional gradient error indicators that are inherently related to the second-order functional derivatives examined in this work.

Consider the second-order, scalar, partial differential equation

$$
\nabla \cdot(p \nabla u)+k^{2} u=g
$$

in the enclosed region $\Omega$ bounded by the surface $S$, with boundary conditions $\left.u\right|_{S_{d}}=u_{d}$ and $\left.(\partial u / \partial n)\right|_{S_{n}}=0$, where

$u \quad$ electromagnetic field unknown for which to be solved;

$p \quad$ material-related parameter equal to $\varepsilon, \mu^{-1}$, or 1 ;

$k \quad$ wave number of the system;

$g$ source function.

If the surface $S$ bounding the entire problem region $\Omega$ is comprised of the union of the Dirichlet and Neumann surfaces, i.e., $S=S_{d}+S_{n}$, then a corresponding functional may be written as

$$
F(u)=\frac{1}{2} \int_{\Omega}\left\{p \nabla u \cdot \nabla u-k^{2} u^{2}+2 g u\right\} d \Omega
$$

for which the true solution to (1) is the admissible function $u$ that renders the above functional $F$ stationary, as explained in [10]. Functional gradient error indicators are closely related to this variational principle used to determine the solution to the finite-element problem [4], [8]. With this type of approach, regions of inferior discretization in a finite-element mesh can be detected and ranked by evaluating the sensitivity of the functional with respect to differential displacements of the geometric nodes. Therefore, by computing the gradients of the functional with respect to vertex positions, it is possible to determine where to improve the discretization. These first-order functional derivative-based error indicators may be computed directly for the functional (2). For example, electromagnetic systems that possess translational or rotational symmetries may be analyzed using 2-D finite-element formulations and in Cartesian problems, where the field solution variation is independent of the coordinate variable $z$, i.e., $u=u(x, y)$, which 
are the $x$ - and $y$-components, respectively, of the first-order functional derivatives for the scalar triangular element with vertex positions $\left(x_{l}, y_{l}\right), l=1,2,3$, may be readily determined from the matrix forms

$$
\frac{1}{2} \mathbf{u}^{T} \mathbf{V u}-b_{l} \frac{k^{2}}{2} \mathbf{u}^{T} \mathbf{B u}+b_{l} \mathbf{u}^{T} \mathbf{B g}
$$

and

$$
\frac{1}{2} \mathbf{u}^{T} \mathbf{W u}-c_{l} \frac{k^{2}}{2} \mathbf{u}^{T} \mathbf{B u}+c_{l} \mathbf{u}^{T} \mathbf{B g}
$$

evaluated over the elements that share the vertex in question. Here, $\mathbf{u}$ and $\mathbf{g}$ are the field solution and the source term vectors, respectively, and the square matrices $\mathbf{V}$ and $\mathbf{W}$ contain the $x$ and $y$ first-order derivative information, respectively, that corresponds to the Laplacian part of the functional for vertex $l(l=1,2,3)$ of a triangular element. The entries of the matrices $\mathbf{V}$ and $\mathbf{W}$ are defined by

$$
\begin{aligned}
& V_{i j}=\frac{1}{4 A^{2}} \sum_{m=1}^{3} \sum_{n=1}^{3} \\
& \cdot\left[2 A \frac{\partial\left(c_{m} c_{n}\right)}{\partial x_{l}}-\left(b_{m} b_{n}+c_{m} c_{n}\right) b_{l}\right] I_{i j m n}
\end{aligned}
$$

and

$$
\begin{aligned}
W_{i j}=\frac{1}{4 A^{2}} & \sum_{m=1}^{3} \sum_{n=1}^{3} \\
\cdot & {\left[2 A \frac{\partial\left(b_{m} b_{n}\right)}{\partial y_{l}}-\left(b_{m} b_{n}+c_{m} c_{n}\right) c_{l}\right] I_{i j m n} }
\end{aligned}
$$

where $A$ is the element area, and $b_{i}$ and $c_{i}$ are geometric parameters related to an element's vertex positions and can be defined as follows with the subscripts progressing modulo 3 :

$$
b_{i}=y_{i+1}-y_{i-1}, \quad \text { and } \quad c_{i}=x_{i-1}-x_{i+1} .
$$

$I_{i j m n}$ is the elemental integral (in homogeneous coordinates) of the product of the derivatives of the $i$ th and $j$ th basis functions, with respect to the $m$ th and $n$th simplex coordinates. The $x$ and $y$ first-derivatives of the wave and source functional terms are given by the second and third terms in each of (3) and (4), respectively, where $B_{i j}$ is the elemental integral (in homogeneous coordinates) of the product of the $i$ th and $j$ th basis functions. Once the gradients of the functional with respect to vertex positions have been computed, they may be used in various ways as error indicators [4]. One simple approach (Type- $A$ ) is to assess a weighted sum of the vertex-based first-order functional derivatives for each element and then use these values to rank the elements for refinement, as explained in [4].

Similarly, second-order functional derivative-based quantities are defined in terms of derivatives with respect to element vertex positions [9]. For example, in 2-D Cartesian problems, the $x$ - and $y$-components, respectively, of the second-order functional derivatives for the scalar triangular element with vertex positions $\left(x_{l}, y_{l}\right), l=1,2,3$ may be readily determined from the matrix forms

$$
\frac{1}{2} \mathbf{u}^{T} \mathbf{P u}, \quad \text { and } \quad \frac{1}{2} \mathbf{u}^{T} \mathbf{Q u}
$$

evaluated over the elements that share the vertex in question. The square matrices $\mathbf{P}$ and $\mathbf{Q}$ contain the $x$ and $y$ secondorder derivative information, respectively, that corresponds to the Laplacian part of the functional for vertex $l(l=1,2,3)$ of a triangular element. The entries of the matrices $\mathbf{P}$ and $\mathbf{Q}$ are defined by

$$
\begin{aligned}
P_{i j}=\frac{1}{4 A^{2}} \sum_{m=1}^{3} & \sum_{n=1}^{3}\left[c_{l} \frac{\partial\left(c_{m} c_{n}\right)}{\partial x_{l}}-b_{l} \frac{\partial\left(b_{m} b_{n}\right)}{\partial y_{l}}\right. \\
+ & \left.2 A \frac{\partial^{2}\left(c_{m} c_{n}\right)}{\partial x_{l}^{2}}\right] I_{i j m n}-\frac{1}{A}\left(b_{l}+c_{l}\right) V_{i j}
\end{aligned}
$$

and

$$
\begin{aligned}
Q_{i j}=\frac{1}{4 A^{2}} & \sum_{m=1}^{3} \sum_{n=1}^{3}\left[b_{l} \frac{\partial\left(b_{m} b_{n}\right)}{\partial y_{l}}-c_{l} \frac{\partial\left(c_{m} c_{n}\right)}{\partial x_{l}}\right. \\
& \left.+2 A \frac{\partial^{2}\left(b_{m} b_{n}\right)}{\partial y_{l}^{2}}\right] I_{i j m n}-\frac{1}{A}\left(b_{l}+c_{l}\right) W_{i j}
\end{aligned}
$$

It should be noted that the "mixed" second-order functional derivative terms with respect to both the $x$ and $y$ element vertex positions are incorporated into the definitions of matrices $\mathbf{P}$ and $\mathbf{Q}$ in (9) and (10). It may also be noted that the partial derivatives of $\left(b_{m} b_{n}\right)$ and $\left(c_{m} c_{n}\right)$ with respect to the element vertex positions, which appear in (5), (6), (9), and (10), can be determined directly from (7) and are given for reference in Table I. Subsequently, the corresponding second-order partial derivatives of these product terms, which appear in (9) and (10), may be readily determined from (7) and Table I: They evaluate to integer constants $(1,-1$, and 0$)$.

As in the first-derivative case [4], the second-order functional derivative formulas are valid for any choice of legitimate finite-element basis functions and may be computed for uniformor mixed-order meshes, as may be required by specific refinement models such as $h-, p-$, or $h p$-adaptive methods. It is interesting to note that the second-order derivatives of the wave and source terms of the functional, with respect to the vertex positions, are zero. This suggests that second-order functional derivative quantities may be most useful for 2-D Laplace systems. It may also be noted that the benefits of combined firstand second-order functional derivative-based local error indicators have been considered in [9]. However, the convergence properties of the practical second-order functional derivative criteria defined by (8) have not been previously investigated and, therefore, are examined in the following sections.

\section{RESULTS}

A simple one-dimensional (1-D) free-space example is presented in order to illustrate the pitfalls that can occur when assessing the finite-element solution convergence with local error estimators. In addition, the practical significance of using second-order functional derivative-based convergence criteria is examined with a 2-D Laplace system for a range of practical adaption models. 
TABLE I

EXPLICIT FORMS OF $\partial\left(b_{m} b_{n_{z}}\right) / \partial y_{l}$ IN TERMS OF $b_{i}$

\begin{tabular}{cccc}
\hline$(m, n) / l$ & 1 & 2 & 3 \\
\hline$(1,1)$ & 0 & $2 b_{1}$ & $-2 b_{1}$ \\
$(1,2),(2,1)$ & $-b_{1}$ & $b_{2}$ & $b_{1}-b_{2}$ \\
$(2,2)$ & $-2 b_{2}$ & 0 & $2 b_{2}$ \\
$(1,3),(3,1)$ & $b_{1}$ & $b_{3}-b_{1}$ & $-b_{3}$ \\
$(2,3),(3,2)$ & $b_{2}-b_{3}$ & $-b_{2}$ & $b_{3}$ \\
$(3,3)$ & $2 b_{3}$ & $-2 b_{3}$ & 0 \\
\hline
\end{tabular}

(a)

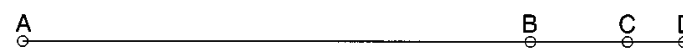

(b)

\begin{tabular}{llll}
$A$ & $B$ & $C$ & $D$ \\
\hline & 0 & 0
\end{tabular}

(c)

$\begin{array}{llll}A & B & C & D\end{array}$

Fig. 1. Three-element discretization for 1-D electrostatic potential analysis of point charge benchmark. (a) Uniform first- and second-order mesh. (b) Globally optimal first-order mesh. (c) Globally optimal second-order mesh. Note that the radial meshes are plotted on a logarithmic scale because of the proximity of some of the nodes to each other near the point charge. The point charge is located at the origin; node A is fixed at $r=0.1 \mathrm{~m}$, node $\mathrm{D}$ is fixed at $r=10 \mathrm{~m}$, and the various positions of nodes $\mathrm{B}$ and $\mathrm{C}$ are specified in Table II.

TABLE II

NumericAl RESUlTS FOR POINT CHARGE BENCHMARK

(a)

\begin{tabular}{ccccc} 
Mesh & 1st-order & 2nd-order & (b) & (c) \\
\hline B Pos. & 3.4000 & 3.4000 & 0.2028 & 0.2717 \\
$d F / d B$ & 2622.3 & 500.17 & 0.0000 & 0.0000 \\
$d^{2} F / d B^{2}$ & 4390.0 & 3424.1 & 3222.2 & 681.75 \\
$E^{-}(B)$ & -2.4865 & 0.6303 & -60.5048 & -7.0431 \\
$E^{+}(B)$ & -0.3738 & -0.3085 & -9.5332 & -7.0431 \\
$C$ Pos. & 6.7000 & 6.7000 & 0.5485 & 1.0153 \\
$d F / d C$ & 13.000 & 0.0899 & 0.0000 & 0.0000 \\
$d^{2} F / d C^{2}$ & 83.410 & 5.2011 & 0.2279 & 0.5303 \\
$E^{-}(C)$ & -0.3738 & -0.0738 & -9.5332 & -0.1336 \\
$E^{+}(C)$ & -0.1398 & -0.0908 & -0.0408 & -0.1336 \\
$F$ Error & $887 \%$ & $340 \%$ & $44.0 \%$ & $10.0 \%$ \\
\hline
\end{tabular}

\section{A. 1-D: Free-Space Point Charge Test System}

This benchmark system was first studied in [7] and is based on the classical free-space point charge model. The objective for this benchmark system is to compute the functional value based on the resolution of a radial neighborhood close to the point charge and spanning a 100-fold decay in electric scalar potential: the point charge, of magnitude $10^{-9} / 9 \mathrm{C}$, is located at the origin, and the two boundaries of the problem domain are set at radial distances of $0.1 \mathrm{~m}$ and $10 \mathrm{~m}$ away from the charge. The primary feature of this system is the rapid field solution variation close to the singularity. This feature is common to many practical devices that contain sharp material corners and has been shown to drastically reduce the convergence rate of the finite-element method. The analysis details and results for the 1-D example, based on resolving the classical point charge benchmark, are given in Fig. 1 and Table I (see also Table II). To focus ideas, functional gradient $(d F / d B$ and $d F / d C)$ [4] and field-discontinuity $\left[E^{ \pm}(B)\right.$ and $\left.E^{ \pm}(C)\right]$ [3] error indicators were evaluated for both uniform and globally optimal first- and

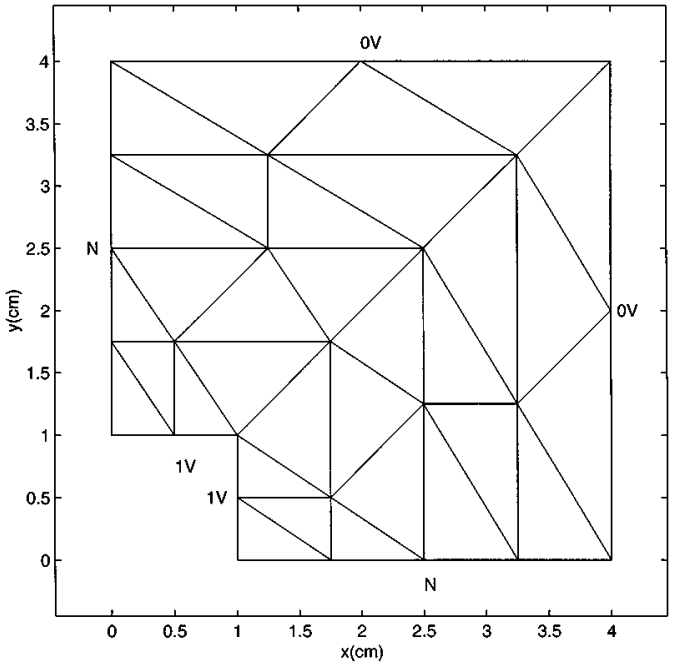

Fig. 2. Laplace benchmark system; initial $h$-mesh (32 triangles).

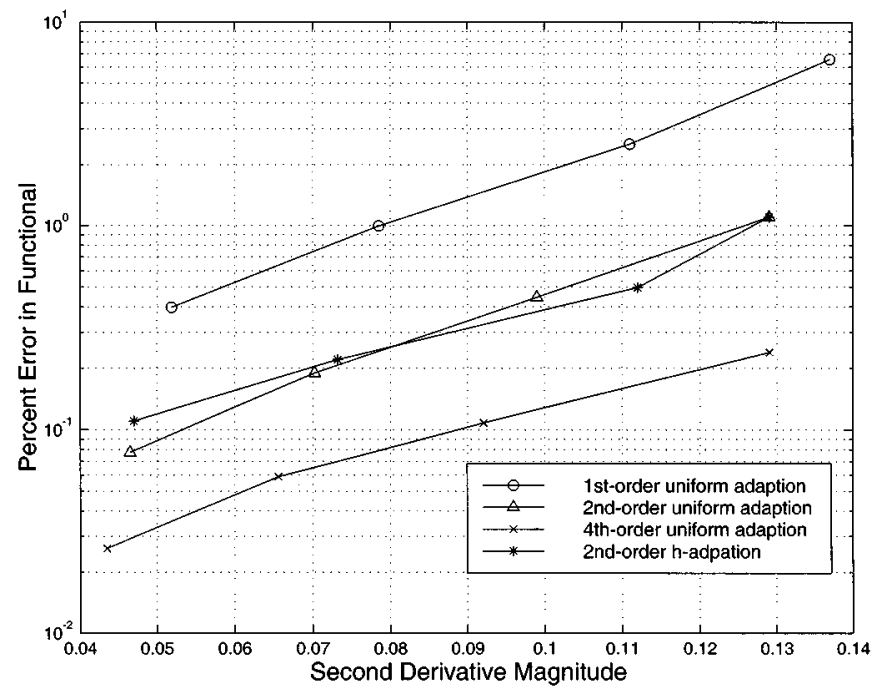

Fig. 3. Second-order $h$-adaption functional derivative convergence.

second-order meshes, based on standard Lagrangian elements. For the uniform discretizations, both the functional gradient and field-discontinuity quantities correctly indicate a large error for the functional value. However, for the optimal discretizations, only the second-order functional derivative correctly suggests poor functional convergence in both cases. The functional gradient error indicators are identically zero, as expected for the optimal meshes, and the field-discontinuity indicator predicts a nonzero functional error only for the first-order case.

\section{B. 2-D Laplace Test System}

The Laplace benchmark system is described by Fig. 2. It is one quarter of a square coaxial line in cross-section - the standard "L" problem. The conductor boundary conditions are 1 $\mathrm{V}$ and $0 \mathrm{~V}$, as indicated, and the symmetry planes are labeled $N$. Second-order functional derivative convergence results are presented in Fig. 3 for first-, second-, and fourth-order uniform $h$-refinements (initial mesh uniformly subdivided to add elements to the discretization). In addition, the second-order $h$-adaption convergence result based on a functional gradient 


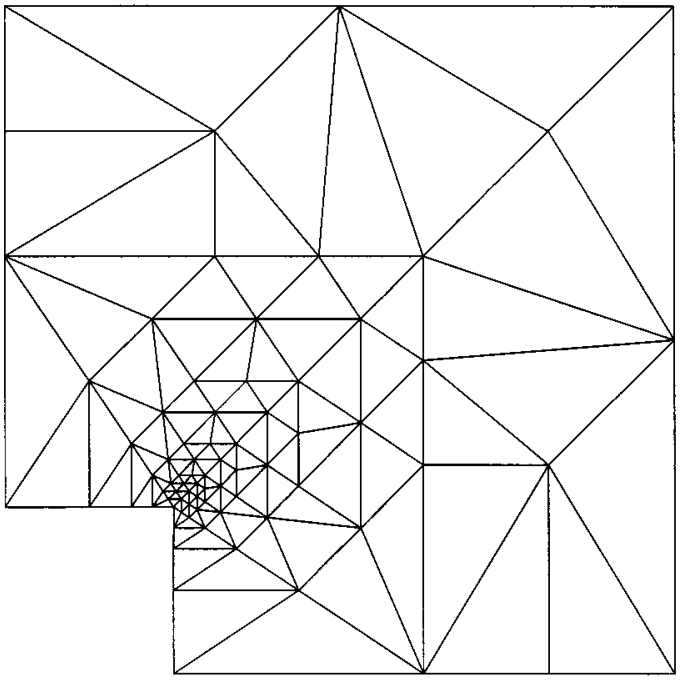

Fig. 4. Example refinement due to $h$-adaption for Laplace system.

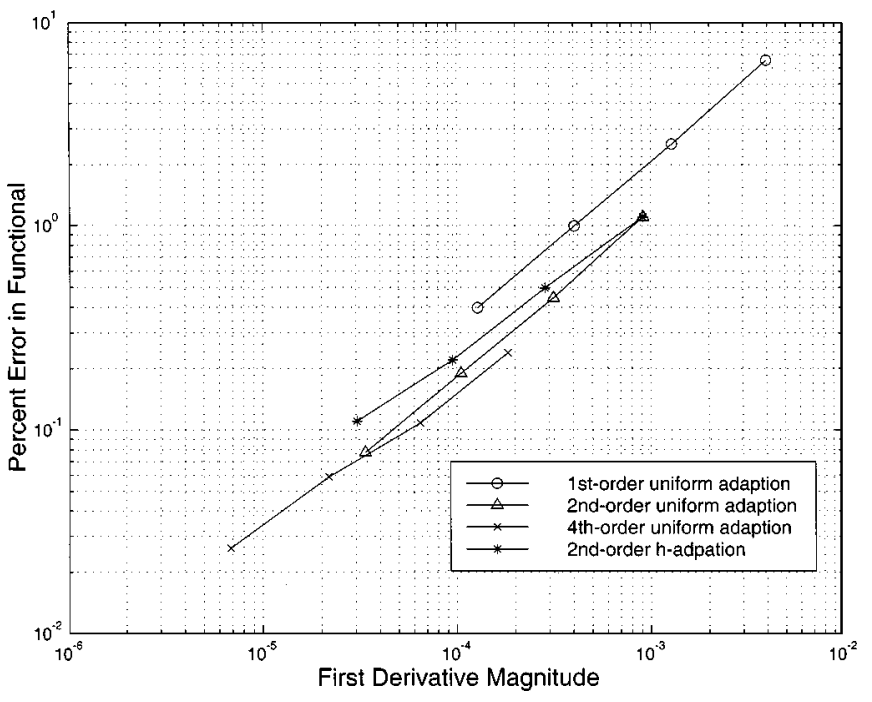

Fig. 5. First-order $h$-adaption functional derivative convergence.

error estimator described earlier (Type-A [4]) is included for comparison. For the latter case, the initial mesh is selectively refined by adding elements to the discretization only in regions of high solution error as determined by the Type- $A$ indicator [4]. An example $h$-adapted mesh is illustrated in Fig. 4 for reference. Note that all the results shown in Fig. 3 correspond to the initial mesh of Fig. 2. In each case, the convergence of the second-order functional derivative is plotted for four refinement iterations. A 50\% increment in the number of DOF per adaptive step was used for the $h$-adaption results. These results sup- port the basic hypothesis that second-order functional derivative-based criteria can be used effectively to assess the convergence of the global solution accuracy for both uniform and $h$-adapted discretizations of Laplace systems. In contrast, the analogous first-order functional derivative convergence results shown in Fig. 5 demonstrate that the global solution accuracy is overestimated by several orders of magnitude. Finally, $p$-adaption (mixed-order meshes) and combined $h p$-adaption convergence results for this benchmark system also corroborate the findings presented in this section (results not shown).

\section{CONCLUSION}

New convergence criteria based on second-order functional derivatives have been proposed and evaluated for scalar 2-D electromagnetic adaptive finite-element analysis. The results for the benchmark systems investigated demonstrate that the second-order derivative criteria may be more reliable for assessing the convergence of the global solution accuracy for arbitrary discretizations of 2-D scalar systems than local error estimators used in practice. These preliminary findings suggest that further theoretical and experimental studies may be useful to clearly establish the practical value of these convergence criteria.

\section{REFERENCES}

[1] P. Fernandes, P. Girdinio, P. Molfino, and M. Repetto, "Local error estimates for adaptive mesh refinement," IEEE Trans. Magn., vol. 24, pp. 299-302, Jan. 1988.

[2] L. Janicke and A. Kost, "Error estimation and adaptive mesh generation in the 2D and 3D finite element method," IEEE Trans. Magn., vol. 32, pp. 1334-1337, May 1996.

[3] J. P. Webb and B. Forghani, "Adaptive improvement of magnetic fields using hierarchal tetrahedral finite elements," IEEE Trans. Magn., vol. 30, pp. 3511-3514, Sept. 1994.

[4] S. McFee and D. Giannacopoulos, "Optimal discretization based refinement criteria for finite element adaption," IEEE Trans. Magn., vol. 32, pp. 1357-1360, May 1996.

[5] L. Janicke and A. Kost, "Convergence properties of the finite element solution,” IEEE Trans. Magn., vol. 35, pp. 1414-1417, May 1999.

[6] C. Geuzaine, B. Meys, P. Dular, and W. Legros, "Convergence of high order curl-conforming finite elements," IEEE Trans. Magn., vol. 35, pp. 1442-1445, May 1999.

[7] D. Giannacopoulos and S. McFee, "Toward optimal $h-p$ adaption near singularities in finite element electromagnetics," IEEE Trans. Magn., vol. 30, pp. 3523-3526, Sept. 1994.

[8] — , "Functional derivatives and optimal discretization based refinement criteria for adaptive finite element analysis with scalar tetrahedra," IEEE Trans. Magn., vol. 35, pp. 1326-1329, May 1999.

[9] S. McFee and D. Giannacopoulos, "The implications of second-order functional derivatives on error estimation in adaptive finite element analysis for electromagnetics," IEEE Trans. Magn., vol. 35, pp. 1330-1333, May 1999.

[10] P. P. Silvester and R. L. Ferrari, Finite Elements for Electrical Engineers, 2nd ed. Cambridge, U.K.: Cambridge Univ. Press, 1990. 